\title{
Predictors of technical adoption and behavioural change to transport energy-saving measures in response to climate change
}

\begin{abstract}
Energy conservation can be achieved through the adoption of technical measures or the changing of one's behaviour. A survey of 201 Malaysian public personnel was conducted to examine the predictors of these two types of transport energy-saving measures in response to climate change. The results indicated that there were significant differences in the relative acceptability of both behavioural measures with respect to gender, level of education, income, knowledge of climate change and attitude. Gender, knowledge of causes of climate change and personal norm were predictors for the acceptability of technical measures, while perceived efficacy and personal norm were the factors that influenced the acceptability of behavioural measures. The results also indicated that distinctions ought to be made between technology adoption and behaviour modifications that require lifestyle changes when assessing pro-environmental intent behaviour. The implications for theory and practice are discussed.
\end{abstract}

Keyword: Transport; Climate change; Energy 